\title{
Myasthenia Gravis Impairment Index: Sensitivity for Change in Generalized Muscle Weakness
}

\author{
Robert H.P. de Meel ${ }^{\mathrm{a}, *}$, Carolina Barnett ${ }^{\mathrm{b}}$, Vera Bril ${ }^{\mathrm{b}, \mathrm{c}}$, Martijn R. Tannemaat ${ }^{\mathrm{a}}$ \\ and Jan J.G.M. Verschuuren ${ }^{\mathrm{a}}$ \\ ${ }^{a}$ Department of Neurology, Leiden University Medical Center, Leiden, The Netherlands \\ ${ }^{\mathrm{b}}$ Department of Medicine, Division of Neurology, University Health Network and University of Toronto, \\ Toronto, Canada \\ ${ }^{\mathrm{c}}$ Institute for Research and Medical Consultations, Imam Abdulrahman Bin Faisal University, \\ Dammam, Saudi Arabia
}

\begin{abstract}
.
Introduction: The recently developed Myasthenia Gravis Impairment Index (MGII) is a promising measure as it has less floor effects and a higher relative efficiency in its responsiveness to treatment effect compared to other MG measures. This study aimed at validating the MGII in a Dutch cohort of MG patients and analyzing the sensitivity of MGII compared to MG-ADL for changes in generalized weakness.

Methods: We analyzed (generalized items of; -gen) MGII, quantitative myasthenia gravis (QMG), Myasthenia Gravis Activities of Daily Living (MG-ADL), EQ-5D visual analog, Myasthenia Gravis Composite (MGC) and ACTIVLIM (an ADL questionnaire focusing on generalized weakness) scores in a prospective cohort of $99 \mathrm{MG}$ patients. We investigated correlations between MGII and other outcome measures. We used a generalized linear model to assess whether MGIIgen had an additional sensitivity on top of MG-ADLgen for changes $(\Delta)$ in QMGgen in individual patients.

Results: MGII had a lower floor effect (4\%) compared to QMG (6\%), MG-ADL (11\%) and MGC (16\%). MGII correlated well with QMG $(\mathrm{r}=0.68)$, MG-ADL $(\mathrm{r}=0.83)$ and MGC $(\mathrm{r}=0.74)$. As expected, the correlations with EQ visual analog and ACTIVLIM were lower $(\mathrm{r}=-0.57$ and -0.48$)$. $\triangle$ MGIIgen had an additional value on top of $\triangle$ MG-ADLgen in the prediction of $\Delta \mathrm{QMGgen}(\mathrm{B}=0.54, p=0.01)$.

Discussion: The MGII score was cross-culturally validated in a Dutch cohort of MG patients. MGII had a higher sensitivity for generalized weakness than MG-ADL.
\end{abstract}

Keywords: Myasthenia gravis, MGII, MG-ADL, QMG, generalized weakness

\section{INTRODUCTION}

Myasthenia gravis (MG) is an autoimmune disease characterized by heterogenous patterns of muscle

\footnotetext{
${ }^{*}$ Correspondence to: Robert H.P. de Meel, Department of Neurology, Leiden University Medical Center, Albinusdreef 2, 2333 ZA Leiden, The Netherlands. Tel.: +31 071 5262118; Fax: +31 071 5266671; E-mail: rhpdemeel@lumc.nl.
}

weakness. Earlier observational studies have suggested that ocular, bulbar or generalized weakness respond differently to different therapies [1-4]. For example, both Barnett et al. and Bhanushali et al. found that ocular symptoms respond relatively well to prednisone treatment. Therefore, an outcome measure's lack of sensitivity for weakness in either of these domains might result in failure to observe 
significant treatment effects. In an earlier study, we found that Myasthenia Gravis Activities of Daily Living (MG-ADL) had a lower sensitivity for changes in generalized weakness [5]. In addition, a prior study reported that patients considered limb weakness as most the disabling symptom, further stressing the importance of a high sensitivity for generalized weakness [6]. The recently developed Myasthenia Gravis Impairment Index (MGII) is a promising measure as it has less floor effects and a higher relative efficiency in its responsiveness to treatment effect compared to other MG measures [7, 8]. The MGII has 22 patient reported and 6 examination items, with 10 items reflecting generalized weakness (appendix). This study aimed at validating the MGII in a Dutch cohort of MG patients and analyzing the sensitivity of the MGII for changes in generalized weakness.

\section{METHODS}

\section{Patients}

We included a prospective cohort of MG patients under treatment at Leiden University Medical Center (LUMC) between 2016 and 2017. The MGII, Quantitative Myasthenia Gravis (QMG), MG-ADL, EQ-5D visual analog (EQ VAS), Myasthenia Gravis Composite (MGC) and ACTIVLIM (acronym of "ACTIVity LIMitations"; an ADL-scale focusing on generalized weakness) scores were recorded in all MG patients who visited the outpatient clinic as part of routine clinical care. The diagnosis of MG was based on a combination of clinically confirmed fluctuating muscle weakness and the presence of serum autoantibodies to the acetylcholine receptor (AChR) or muscle-specific kinase (MuSK). Seronegative myasthenia gravis (SNMG) was defined as fatigable muscle weakness in combination with abnormal decrement (at least 10\%) during low-frequency repetitive nerve stimulation, increased jitter in single-fiber EMG testing or a positive neostigmine test [9]. This study was approved by the Medical Ethics Boards of the Leiden University Medical Center. All patients provided informed, written consent prior to study participation.

\section{Cross-cultural translation MGII}

In order to translate the MGII from English to Dutch whilst taking into account cultural differences, we used the back-translation method. The original English version of the MGII was translated to Dutch by a physician at our center. This Dutch version of the MGII was subsequently back-translated to English by independent translators, blinded to the original measure. The back-translation was then compared to the original, and items with major differences were revised [10].

\section{Subdomains outcome variables}

The first 5 items of the QMG constitute the oculobulbar domain (QMGob) and the remaining 8 items constitute the generalized domain (QMGgen). We considered QMGgen to be representative of objective generalized muscle weakness [5]. The first 3 and last 2 items of the MG-ADL constitute the oculobulbar domain (MG-ADLob) and the 4th until the 6th items constitute the generalized domain (MGADLgen). The first 15 items of the questionnaire and the first 3 physical examination items of MGII constitute the oculobulbar domain (MGIIob) and the last 7 items of the questionnaire and the last 3 physical examination items constitute the generalized domain (MGIIgen).

\section{Statistical analysis}

To reproduce earlier reported construct validity findings of the MGII, we analyzed correlations between MGII and other outcome measures [8]. We investigated whether there were significantly higher generalized scores (MGIIgen, MG-ADLgen and QMGgen) in patients with generalized MG compared to ocular MG patients with an unpaired $t$-test. We used a generalized linear model to assess whether MGIIgen had an additional sensitivity on top of MGADLgen for changes $(\Delta)$ in QMGgen in individual patients. Results are expressed as B coefficients with 95\% confidence intervals (CI), which reflect the additional value of MGII in the prediction of QMGgen compared to MG-ADLgen alone. The regression line shows the degree in which MGIIgen can compensate for the mismatch between observed and predicted QMGgen. $P$-values $<0.05$ were considered significant. Statistical analyses were performed using SPSS version 23 (IBM Corp., Armonk, NY).

\section{RESULTS}

We included 99 consecutive patients with MG. Of this group, 10 patients had a second visit with all assessments. Demographic and clinical baseline characteristics of all patients are shown in Table 1. 
Table 1

Demographic and clinical baseline characteristics

\begin{tabular}{|c|c|}
\hline MG patients $\mathrm{N}=99$ & \\
\hline Age, y & $57.4 \pm 18.1$ \\
\hline Age at onset, y & $45.5 \pm 20.0$ \\
\hline \multicolumn{2}{|l|}{ Gender } \\
\hline Male & $34(34)$ \\
\hline Female & $65(66)$ \\
\hline \multicolumn{2}{|l|}{ Antibodies } \\
\hline AChR+ & $71(72)$ \\
\hline MuSK+ & $8(8)$ \\
\hline Seronegative & $20(20)$ \\
\hline \multicolumn{2}{|l|}{ Phenotype } \\
\hline Oculobulbar & $24(24)$ \\
\hline Generalized & $75(76)$ \\
\hline \multicolumn{2}{|l|}{ Thymectomy } \\
\hline Yes, with thymoma & $8(8)$ \\
\hline Yes, without thymoma & $7(7)$ \\
\hline No & $84(85)$ \\
\hline QMG & $6.7 \pm 4.8$ \\
\hline QMGob & $2.2 \pm 2.5$ \\
\hline QMGgen & $4.4 \pm 3.7$ \\
\hline MG-ADL & $4.8 \pm 3.5$ \\
\hline MG-ADL $o b$ & $3.1 \pm 2.6$ \\
\hline MG-ADLgen & $1.8 \pm 1.7$ \\
\hline MGII & $19.5 \pm 15.0$ \\
\hline MGII $o b$ & $12.0 \pm 10.6$ \\
\hline MGIIgen & $7.5 \pm 7.1$ \\
\hline MGC & $7.0 \pm 6.5$ \\
\hline EQ VAS & $67.7 \pm 19.4$ \\
\hline ACTIVLIM & $3.6 \pm 2.1$ \\
\hline
\end{tabular}

Baseline characteristics of 99 patients with MG included in this study. Data are presented as number of patients (\%) for categorical variables and as mean \pm SD for continuous variables.

\section{Cross-cultural translation}

In the back translated version of the MGII, only the item assessing leg weakness with effort was different. The original item is phrased as weakness after walking a certain number of blocks. In the Netherlands, blocks are not commonly used, so blocks were converted to meters, and we kept this wording as it is culturally appropriate. All other items are a literal translation of the original.

\section{Construct validity}

The mean total MGII score was $19.5 \pm 15.0$, the mean oculobulbar score $12.0 \pm 10.6$, and the mean generalized subscore 7.5 7.1. MGII had a lower floor effect (4\%) compared to QMG (6\%), MGADL (11\%) and MGC (16\%). Generalized MG patients $(\mathrm{N}=75)$ had a significantly higher MGIIgen than oculobulbar MG patients $(\mathrm{N}=24)(8.6 \pm 6.9$ and $4.3 \pm 6.7 ; p=0.01$, respectively). There were non-significant differences between generalized and
Table 2

Correlation between MGII and other measures

\begin{tabular}{lcc}
\hline Comparison measure & Pearson $\mathrm{r}(95 \%$ CI $)$ & $p$ Value \\
\hline QMG & $0.68(0.56$ to 0.77$)$ & $<0.001$ \\
MG-ADL & $0.83(0.76$ to 0.88$)$ & $<0.001$ \\
MGC & $0.74(0.64$ to 0.82$)$ & $<0.001$ \\
EQ VAS & $-0.57(-0.69$ to -0.42$)$ & $<0.001$ \\
ACTIVLIM & $-0.48(-0.62$ to -0.31$)$ & $<0.001$ \\
\hline
\end{tabular}

Correlations between MGII and other outcome measures are shown. With these correlations construct validity was tested. In ACTIVLIM and EQ VAS higher scores indicate a higher activity level or quality of life.

oculobulbar MG patients when comparing QMGgen $(4.7 \pm 3.9$ and $3.6 \pm 2.9 ; p=0.14$, respectively) and MG-ADLgen $(1.7 \pm 1.6$ and $2.2 \pm 2.0 ; p=0.25$, respectively). Table 2 shows the correlations between MGII and other outcome measures.

\section{Responsiveness to change in generalized muscle weakness}

The correlations between $\triangle$ MGIIgen and $\triangle$ QMGgen or between $\triangle$ MG-ADLgen and $\triangle$ QMGgen were both significant $(\mathrm{B}=0.78$, $p<0.001$, and $\mathrm{B}=1.50, p=0.01$, respectively). The MGIIgen score has a range of $0-31$, whereas the MG-ADLgen has a range of $0-9$, which explains why $\Delta$ MGIIgen has a more significant correlation compared to $\triangle \mathrm{MG}$-ADLgen notwithstanding the lower B coefficient. The mean deviation of $\Delta \mathrm{MG}-$ ADLgen from the predicted values of $\triangle \mathrm{QMGgen}$ was 3.5 \pm 2.5. $\triangle$ MGIIgen had an additional value on top of $\triangle \mathrm{MG}$-ADLgen in the prediction of QMGgen $(\mathrm{B}=0.54, p=0.01$; Fig. 1$)$.

\section{DISCUSSION}

The MGII scale was cross-culturally validated in a cohort of 99 Dutch MG patients. $\Delta$ MGIIgen had a significantly higher sensitivity for changes in generalized weakness than $\triangle \mathrm{MG}$-ADLgen. Similarly to the findings of Barnet et al., the MGIIgen subscore was significantly higher in generalized patients compared to oculobulbar patients. In contrast, MG-ADLgen was not capable of differentiating oculobulbar from generalized patients.

The correlation of MGII to both other MG-specific measures and non-specific measures were all within the range $(r=0.6-0.8$ and $r=0.4-0.7$, respectively $)$ as hypothesized by the developers of the MGII. Only the MG-ADL was slightly above the hypothesized range (0.83), which also was the case in the previous study 


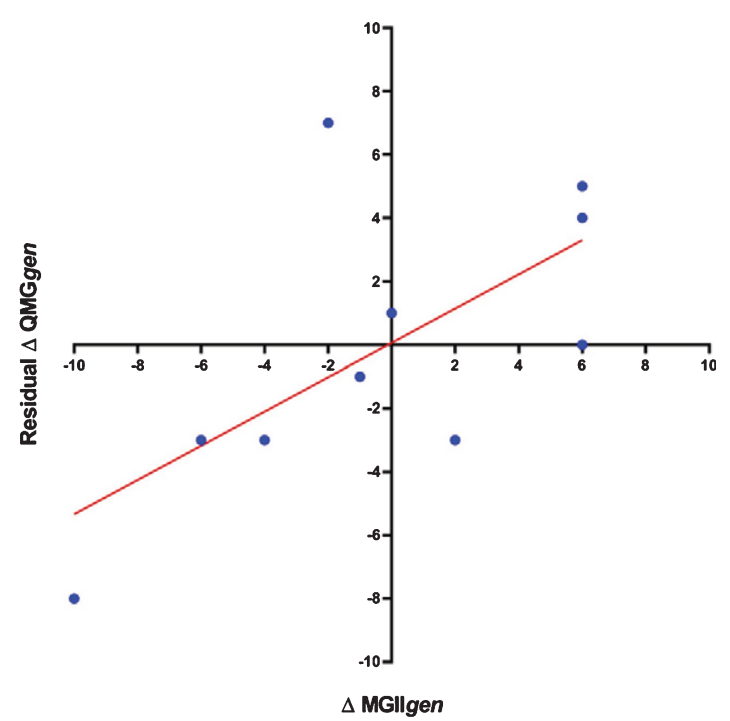

\begin{tabular}{|c|c|c|c|c|}
\hline $\begin{array}{l}\text { Correlation } \\
\text { AMGHgen to: }\end{array}$ & $\begin{array}{l}\text { No. of } \\
\text { patients }\end{array}$ & B coefficient & 95\% CI & $p$ Value \\
\hline Residual $\Delta$ QMGgen & 10 & 0.54 & $0.15 ; 0.92$ & 0.01 \\
\hline
\end{tabular}

Fig. 1. Analysis of the additional sensitivity of $\triangle$ MGIIgen on top of $\Delta \mathrm{MG}$-ADLgen for $\Delta \mathrm{QMG} g e n$ in 10 patients with two visits. The dots show the $\Delta \mathrm{QMGgen}$ residual for each individual patient. The $\Delta$ QMGgen residual is the difference between observed and predicted $\triangle \mathrm{QMGgen}$ based on the $\triangle \mathrm{MG}$-ADL score of that patient. The $\mathrm{B}$ coefficient (slope) shows the degree in which $\Delta \mathrm{MGII}$ correlates with the residual of $\Delta \mathrm{QMGgen}$. The significant correlation implies that $\Delta \mathrm{MGII}$ can to some degree compensate for the mismatch between observed and predicted $\Delta \mathrm{QMGgen}$ and therefore has an additional sensitivity for generalized muscle weakness on top of $\triangle \mathrm{MG}-\mathrm{ADL}$.

of Barnett et al. The floor effects found in this study and in that of Barnett et al. were (almost) identical for MGII (4\% and 5\%, respectively) and for MGC (16\% in both studies). The floor effect of MG-ADL found in our study was lower $(11 \%)$ than that found in the study of Barnet et al. (22\%). Nonetheless, the floor effect of MGII was shown to be clearly lower than that of MG-ADL.

Limitations of this study include the single center of inclusion and our study population within a tertiary referral center that may not fully reflect the total MG population due to a referral bias. Moreover, it might be argued that QMGgen is not a 'gold standard' regarding changes in objective generalized muscle weakness. In this study, we chose to use QMGgen as reference for generalized weakness as the QMG score is the best-known structured way of quantifying muscle weakness in MG and has a high focus on generalized weakness (8 out of 13 items).

Weakness in MG is not 'unidimensional' and muscle groups do not respond equally to different therapies. Outcome measures should therefore be sensitive to changes in both oculobulbar and generalized weakness. This study has shown that in addition to the high construct validity of the MGII in general, this outcome measure is more sensitive to changes in generalized weakness than the MG-ADL. We recommend including MGII in future clinical trials.

\section{DISCLOSURE OF CONFLICT OF INTEREST}

Dr. Barnett is the primary developer of the MGII. She has received grant support from Octapharma and Grifols. She also has acted as consultant for UCB, Alexion and Akcea.

All other authors have no conflicts of interest to declare.

\section{SUPPLEMENTARY MATERIAL}

The appendix available in the electronic version of this article: https://dx.doi.org/10.3233/JND200484.

\section{REFERENCES}

[1] Lewis RA. Myasthenia gravis: new therapeutic approaches based on pathophysiology. Journal of the Neurological Sciences. 2013;333:93-8.

[2] de Meel RHP, Verschuuren J, Tannemaat MR. Distinct representation of muscle weakness in QMG and MG-ADL. The Lancet Neurology. 2018;17:204-5.

[3] Bhanushali MJ, Wuu J, Benatar M. Treatment of ocular symptoms in myasthenia gravis. Neurology. 2008;71:133541.

[4] Barnett TC, Bril V, Davis AM. Performance of individual items of the quantitative myasthenia gravis score. Neuromuscular Disorders : NMD. 2013;23:413-7.

[5] de Meel RHP, Raadsheer WF, van Zwet EW, Verschuuren J, Tannemaat MR. Sensitivity of MG-ADL for generalized weakness in myasthenia gravis. European Journal of Neurology. 2019;26:947-50.

[6] Abraham A, Breiner A, Barnett C, Katzberg HD, Bril $\mathrm{V}$. The utility of a single simple question in the evaluation of patients with myasthenia gravis. Muscle \& Nerve. 2018;57:240-4.

[7] Barnett C, Bril V, Kapral M, Kulkarni AV, Davis AM. Myasthenia Gravis Impairment Index: Responsiveness, meaningful change, and relative efficiency. Neurology. 2017;89:2357-64.

[8] Barnett C, Bril V, Kapral M, Kulkarni A, Davis AM. Development and validation of the Myasthenia Gravis Impairment Index. Neurology. 2016;87:879-86.

[9] Meriggioli MN, Sanders DB. Autoimmune myasthenia gravis: emerging clinical and biological heterogeneity. The Lancet Neurology. 2009;8:475-90.

[10] Sperber AD. Translation and validation of study instruments for cross-cultural research. Gastroenterology. 2004;126:S124-8. 